\title{
Left ventricular mass and systolic function in children with chronic kidney disease - comparing echocardiography with cardiac magnetic resonance imaging
}

\author{
Raoul Arnold ${ }^{1}$ - Daniel Schwendinger ${ }^{2} \cdot$ Sabine Jung $^{3} \cdot$ Martin Pohl $^{5} \cdot$ Bernd Jung $^{6} \cdot$ \\ Julia Geiger $^{4} \cdot$ Charlotte Gimpel $^{5}$ (1)
}

Received: 22 June 2015 /Revised: 14 August 2015 / Accepted: 17 August 2015 / Published online: 5 September 2015

(C) IPNA 2015

\begin{abstract}
Background Increased left ventricular mass (LVM) is an important risk marker of uremic cardiovascular disease. Calculation of LVM by echocardiography (Echo) relies on geometric assumptions and in adults on hemodialysis overestimates LVM compared to cardiac magnetic resonance (CMR). We compare both techniques in children with chronic kidney disease (CKD).

Methods Concurrent Echo and CMR was performed in 25 children with CKD (14 after kidney transplantation) aged $8-17$ years.

Results Compared to normal children, CMR-LVM was increased (standard deviation score (SDS) $0.39 \pm 0.8(p=$
\end{abstract}

Electronic supplementary material The online version of this article (doi:10.1007/s00467-015-3198-z) contains supplementary material, which is available to authorized users.

Charlotte Gimpel

charlotte.gimpel@uniklinik-freiburg.de

1 Department of Pediatric Cardiology, Center for Pediatrics and Adolescent Medicine, University Hospital Heidelberg, Heidelberg, Germany

2 Hospital for Children, Stadtspital Triemli, Zurich, Switzerland

3 Division of Nuclear Medicine, University Hospital Freiburg, Freiburg, Germany

4 Department of Radiology, University Children's Hospital Zurich, Zurich, Switzerland

5 Center for Pediatrics and Adolescent Medicine, University Hospital Freiburg, Freiburg, Germany

6 Institute of Diagnostic, Interventional and Pediatric Radiology, Inselspital, Bern, Switzerland
$0.03)$ ), stroke volume and cardiac output decreased (SDS $-1.76 \pm 1.1, p=0.002$ and $-1.11 \pm 2.0, p=0.001)$. CMR-LVM index but not Echo-LVMI correlated to future glomerular filtration rate $(\mathrm{GFR})$ decline $(r=-0.52, p=0.01)$. Mean Echo-LVM was higher than CMR-LVM (117 \pm 40 vs. $89 \pm$ $29 \mathrm{~g}, p<0.0001)$, with wide limits of agreement ( -6.2 to $62.8 \mathrm{~g}$ ). The Echo-CMR LVM difference increased with higher Echo-LVMI $(r=0.77, p<0.0001)$. Agreement of classifying left ventricular hypertrophy was poor with Cohen's kappa of 0.08. Mean Echo and CMR-ejection fraction differed by $1.42 \%$ with wide limits of agreement ( -12.6 to $15.4 \%)$. Conclusions Echo overestimates LVM compared to CMR, especially at higher LVM. Despite this, CMR confirms increased LVM in children with CKD. Only CMR-LVMI but not Echo-LVMI correlated to future GFR decline.

Keywords Left ventricular hypertrophy - Ejection fraction . Echocardiography $\cdot \mathrm{CMR} \cdot$ Chronic renal failure $\cdot$ Adolescents

\section{Introduction}

Cardiovascular mortality is the leading cause of death in adult patients with chronic kidney disease and end-stage renal failure. Left ventricular mass (LVM) is an established risk marker for prediction of mortality. Children with chronic kidney disease (CKD) also show features of beginning cardiovascular disease such as left ventricular hypertrophy (LVH) [1, 2], alterations of systolic [3] and diastolic function [4, 5], increased intima media thickness $[6,7]$, and stiffening of the aorta and peripheral vessels [8], even though other risk factors such as diabetes or smoking are normally absent. Despite the very low cardiovascular mortality in this age group, early detection of 
cardiovascular abnormalities is still important in evaluating the risk of and potentially prevent future cardiovascular disease.

Echocardiography (Echo) offers a well-established and non-invasive technique for cardiac examination. LVM can be estimated by the American Society of Echocardiography (ASE) formula, which is validated by autopsy in adults [9]. LVM-index (LVMI) to body height to the power of 2.7 is preferred over normalization to body surface area (BSA) as the latter underestimates LVH in overweight patients [10]. LVMI cut-offs are also suitable for children over the age of 9 years, however, in younger patients LVMI is still heightdependent. For children, normal values for LVM and LVMI are available by Khoury [11], which for older children are quite similar to the 95th percentile of LVMI found by the group of de Simone [2, 12].

Several problems remain though: the ASE formula has not been validated in children and because it uses squares of several measurements, it is easily distorted by measurement errors. Additionally, it is less suitable for patients with altered geometry, which may sometimes be the case in fluidoverloaded patients. Application of different echo reference values can result in large differences in the number of children classified to have LVH and thus raises doubt whether this method is reliable in children with chronic kidney disease $[12,13]$.

Cardiac magnetic resonance imaging (CMR) offers an alternative for measuring LVM, which is not dependent on normal cardiac geometry, as heart contours are individually traced. CMR has higher inter-reader and inter-study reproducibility for measuring LVM than echocardiography [14, 15]. Echo and CMR are both predictive of mortality but no headto-head comparison has been performed in any patient group [16]. Two studies have employed CMR in children with CKD and have been able to partially reproduce echocardiographic findings $[17,18]$. However, when compared to CMR, echocardiography substantially overestimates LVM in adult hemodialysis patients $[19,20]$, but this has not been examined in children. Therefore, the aim of this study was to assess the agreement of Echo and CMR in quantifying both left ventricular mass and functional parameters in children with chronic kidney disease.

\section{Patients and methods}

\section{Patients}

Children and adolescents with chronic kidney disease stage 3 or higher (i.e., with an estimated glomerular filtration rate (GFR) below $60 \mathrm{ml} / \mathrm{min}^{*} 1.73 \mathrm{~m}^{2}$ ) and/or who had undergone renal transplantation were eligible for the study. The presence of structural anomalies of the heart, cardiac valves or great vessels, cardiomyopathies, a pacemaker, or other metallic foreign bodies were exclusion criteria.

\section{Imaging techniques}

Cardiac magnetic resonance scans without contrast medium were performed on a 1.5-Tesla scanner (TIM-Symphony, Siemens, Erlangen, Germany). For the measurement of LVM ECG-gated steady-state free precession (SSFP) cine images were taken of at least ten contiguous slices along the short axis of the heart (parallel to the plane of the atrioventricular valves) [21] using the following parameters: Echo time $1.25 \mathrm{~ms}$, repetition time $3 \mathrm{~ms}$, field of view $270 \mathrm{~mm}$, slice thickness $8 \mathrm{~mm}$, temporal resolution $24.8 \mathrm{~ms}$, spatial resolution 1.5-2 mm. End-diastolic epi- and endocardial contours of the left ventricle were traced manually on each slice and a software (Argus, Siemens) used to calculate the end-diastolic left ventricular muscle volume (not including the papillary muscles) by multiplication of the cross-sectional area by the distance between slices. Further multiplication of the muscle volume by the specific muscle weight yields ventricular mass [22]. Stroke volume (SV) was measured as the difference between the end-systolic and end-diastolic volume (ESV and EDV), and the ejection fraction (EF) calculated as stroke volume/end-diastolic volume.

On the same day, 2D-guided M-Mode echocardiography was performed according to the standards of the American Society of Echocardiography [23] by an experienced examiner on a Vivid 7 Dimension cardiovascular ultrasound system (GE Healthcare, Milwaukee, WI, USA). Electronic calipers were used to measure left ventricular end-diastolic diameter, diastolic posterior wall thickness and interventricular septal thickness in order to calculate left ventricular mass according to the American Society of Echocardiography (ASE) formula by Devereux [9]. EF was also calculated based on M-mode measurements. For quality control, a number of examinations were checked by a second examiner.

\section{Clinical measurements}

Clinical examination, anthropometric measurements, and clinic blood pressure were usually taken on the same day as the imaging procedures. The following blood parameters were also measured: serum creatinine, cystatin $\mathrm{C}$, bound urea nitrogen, standard blood count and electrolytes, parathyroid hormone, 25-OH-vitamin $\mathrm{D}, \mathrm{N}$-terminal pro brain natriuretic peptide (pro BNP), renin, and aldosterone (in supine position). Spot urines were used to quantify proteinuria as total protein/creatinine ratio. Ambulatory 24-h blood pressure measurements (ABPM) were taken every 15 min during the day and every $30 \mathrm{~min}$ during the night using Spacelabs 9,0207 monitors (Spacelabs Healthcare, Snoqualmie, WA, USA). 
Data on kidney function, blood pressure control, and medication over 6 months preceding the study day were taken from clinical records. From the time of study, serum creatinine, BUN, and body height were recorded prospectively until start of renal replacement therapy $(n=3)$ or transfer to adult unit $(n=8)$. Two patients were lost to follow-up and one excluded due to rapid loss of transplant function due to noncompliance. The remaining 22 patients were followed up for $36.3 \pm 9.8$ months (range, 12-49 months).

\section{Normal values}

For Echos, LVH was classified as LVMI $>95$ th percentile for age according to the largest available reference population presented by Khoury et al. [11]. Incidentally, use of these age- and height-dependent percentiles resulted in identical patients being classified as having LVH as the use of the age- and height-independent cut-off found by the group of de Simone $[2,12]$ (where LVMI above $38.6 \mathrm{~g} / \mathrm{m}^{2.7}$ is considered hypertrophic). In addition, a relative wall thickness (RWT $=$ posterior wall thickness/left ventricular end diastolic diameter) greater than 0.375 was used to distinguish concentric from eccentric hypertrophy and concentric remodeling from normal cardiac structure [24].

For normal ranges of cardiac mass on CMR using SSFP, normal populations have been examined by Buechel et al. [25], Robbers-Visser et al. [26], as well as Sarikouch et al. [27]. The latter two have been pooled by Kawel-Boehm [28] and are used here, as they represent the largest cohort and use an identical technique (e.g., papillary muscles not included in volumes). We define LVH as LVM/BSA above the 95th percentile (mean $+1.65 \times$ standard deviation).

ABPM standard deviation scores of blood pressure were calculated using normal values by Wühl et al. [29], while a large up-to-date German reference study (KIGGS) was used for normal values of clinic blood pressure and anthropometric measurements $[30,31]$.

\section{Statistics}

Before the start of the study, a power analysis estimated that a sample of about 15 children would be large enough to detect significant differences of LVMI from the norm. Group comparisons were made using the Chi-square test for categorical variables and Student's $t$ test for continuous variables. The Bland-Altman method [32] was used to compare CMR and Echo measurements. To compare classification of LVH, Cohen's kappa was used [33], where a value of 0 signifies that the agreement between two methods is only as good as chance and 1 that they agree perfectly (negative values are possible and indicate agreement worse than expected by chance). Values below 0.2 are generally regarded as poor agreement and only above 0.61 as good or above 0.81 as very good [34]. Correlations are quantified by giving Pearson's correlation coefficient. Throughout, $p$ values less than 0.05 were considered significant. Statistical analyses were performed using SAS V9.4 (SAS Institute, Cary, NC, USA).

\section{Results}

\section{Patient characteristics}

Twenty-eight patients consented to take part in the study. Two patients (a 13-year-old female and a 17-year-old male) withdrew due to unexpected claustrophobia in the scanner. One CMR study was not analyzable due to data storage problems. Of the remaining 25 children, 11 were males ( $44 \%$ ), 11 had chronic kidney disease (44\%), and 14 had received a renal transplant (56\%); none were on dialysis. Further patient characteristics are shown in Table 1. CKD was due to renal dysplasia or agenesis (with or without urinary tract malformations) in nine patients, congenital or acquired glomerulopathy in nine, nephronophthisis in four, and other causes in three patients. Small proteinuria (protein/creatinine ratio $0.2-1 \mathrm{~g} / \mathrm{g}$ in spot urine) was present in six patients and large proteinuria (protein/creatinine ratio $>1 \mathrm{~g} / \mathrm{g}$ ) in five children, none of whom were nephrotic.

Mean blood pressure was mildly elevated (see Table 1), however this was only possible with antihypertensive medication in 21 patients ( $85 \%$ ) (four patients received one agent, ten received two agents, and seven received three or more antihypertensive medications). The most commonly used drugs were ACE inhibitors $(n=14)$, amlodipine $(n=13)$, metoprolol $(n=11)$, angiotensin receptor blocker $(n=6)$, and diuretics $(n=3)$. Uncontrolled systolic hypertension was revealed by ABPM in four patients, while none had diastolic hypertension on ABPM. Three of these four had normal clinic blood pressure, while seven children had white coat hypertension.

Renal anemia was treated with iron supplements in eight patients and with additional erythropoiesis-stimulating agents in five. Overt anemia (hemoglobin below $11 \mathrm{~g} / \mathrm{l}$ ) was present in five patients.

\section{Echocardiography}

Findings on standard echocardiography are shown in Table 2. Left ventricular hypertrophy was present in eight children (32\%), with exact agreement between the classification according to normal values by Khoury [11] and Matteuci [2]. Hypertrophy was concentric in seven and eccentric in one child ( $28 \%$ and $4 \%$ ), while 12 of 17 without hypertrophy had concentric remodeling ( $48 \%$ overall). LVM for BSA was above the 95th percentile in seven children (of whom six also 
Table 1 Patient characteristics

\begin{tabular}{lllll}
\hline & Mean & SD & Minimum & Maximum \\
\hline Age (years) & 13.72 & 2.85 & 8.3 & 17.7 \\
Height (cm) & 154.3 & 16.8 & 124 & 185 \\
Weight (kg) & 47.8 & 15.2 & 25.3 & 75.2 \\
Body surface area* $\left(\mathrm{m}^{2}\right)$ & 1.43 & 0.3 & 0.96 & 1.92 \\
Height SDS & -0.79 & 1.02 & -2.68 & 1.65 \\
BMI SDS & -0.19 & 0.8 & -1.67 & 1.39 \\
Systolic/diastolic BP (mmHg) & $122 / 70$ & $11 / 9$ & $98 / 55$ & $144 / 90$ \\
Systolic/diastolic BP SDS & $0.97 / 0.57$ & $0.9 / 1.2$ & $-0.4 /-1.3$ & $3.1 / 3.35$ \\
Systolic/diastolic 24 h BP (mmHg) & $116 / 68$ & $8.1 / 5$ & $101 / 56$ & $129 / 76$ \\
Systolic/diastolic 24 h BP SDS & $0.47 / 0.08$ & $1.1 / 1.0$ & $-0.9 /-2.3$ & $2.4 / 1.4$ \\
Creatinine ( $\mu$ mol/l) & 151 & 95 & 69 & 473 \\
Cystatin C (mg/l) & 1.7 & 0.7 & 0.8 & 3.6 \\
eGFR (creatinine**) (ml/min/1.73 $\left.\mathrm{m}^{2}\right)$ & 62 & 26 & 14 & 113 \\
eGFR (cystatin C***) (ml/min/1.73 $\left.\mathrm{m}^{2}\right)$ & 60 & 24 & 21 & 116 \\
Hemoglobin (g/dl) & 12.4 & 1.6 & 8.4 & 14.4 \\
\hline
\end{tabular}

$S D$ standard deviation, $S D S$ standard deviation score, $B P$ blood pressure, $B M I$ body mass index, $e G F R$ estimated glomerular filtration rate

*DuBois formula

**Schwartz formula [35]

***formula according to Filler [36]

had an increased LVMI, but one was normal according to Khoury [11]).

\section{CMR}

Left ventricular dimensions and functional parameters measured on CMR are shown in Table 3. CMR-LVM was

Table 2 Echocardiographic measurements, left ventricular dimensions, and systolic function in 25 children with chronic kidney disease and/or after renal transplantation

\begin{tabular}{|c|c|c|c|c|}
\hline & Mean & SD & Minimum & Maximum \\
\hline \multicolumn{5}{|c|}{ Measurements of cardiac dimensions } \\
\hline LVEDD (mm) & 41.6 & 5.0 & 33 & 51 \\
\hline LVPWT (mm) & 8.92 & 2.06 & 6 & 15 \\
\hline IVST (mm) & 8.72 & 2.30 & 5 & 13 \\
\hline \multicolumn{5}{|c|}{ Calculated cardiac dimensions } \\
\hline $\operatorname{LVM}(\mathrm{g})$ & 117.0 & 39.7 & 62 & 207 \\
\hline $\operatorname{LVMI}\left(\mathrm{g} / \mathrm{m}^{2.7}\right)$ & 35.6 & 7.3 & 23 & 48 \\
\hline LVM/BSA $\left(\mathrm{g} / \mathrm{m}^{2}\right)$ & 81.0 & 17.6 & 56 & 113 \\
\hline RWT & 0.43 & 0.09 & 0.30 & 0.69 \\
\hline \multicolumn{5}{|c|}{ Parameters of systolic function } \\
\hline $\mathrm{EF}(\%)$ & 64.7 & 6.6 & 52 & 75 \\
\hline FS $(\%)$ & 36.4 & 8.1 & 26 & 68 \\
\hline
\end{tabular}

LVEDD left ventricular end diastolic diameter, $L V P W T$ left ventricular posterior wall thickness, IVST interventricular septal thickness, $L V M$ left ventricular mass, $L V M I$ LVM index, $R W T$ relative wall thickness, $E F$ ejection fraction, $F S$ endocardial fractional shortening, $S D$ standard deviation increased significantly (i.e., SDS significantly above 0 , see Table 3), while EDV, SV, and cardiac index were decreased from normal. However, LV hypertrophy was present in

Table 3 Measurements and SDS scores of cardiac dimensions and systolic function on cardiac magnetic resonance in 25 children with chronic kidney disease and/or after renal transplantation

\begin{tabular}{|c|c|c|c|c|c|}
\hline & Mean & $\mathrm{SD}$ & Minimum & Maximum & $p$ value \\
\hline \multicolumn{6}{|c|}{ Measurements of cardiac dimensions } \\
\hline $\operatorname{LVM}(\mathrm{g})$ & 88.7 & 29.0 & 47 & 154 & \\
\hline $\operatorname{LVM}_{\mathrm{BSA}}\left(\mathrm{g} / \mathrm{m}^{2}\right)$ & 61.1 & 10.6 & 43 & 84 & \\
\hline LV EDV (ml) & 103 & 31.9 & 50 & 181 & \\
\hline LV ESV (ml) & 38.7 & 16.4 & 16 & 85 & \\
\hline \multicolumn{6}{|c|}{ Parameters of systolic function } \\
\hline $\mathrm{EF}(\%)$ & 63.3 & 8.4 & 39.8 & 75.2 & \\
\hline $\mathrm{SV}(\mathrm{ml})$ & 64.8 & 20.2 & 34.2 & 122.4 & \\
\hline $\mathrm{CI}\left(1 / \mathrm{min} / \mathrm{m}^{2}\right)$ & 3.32 & 0.9 & 1.69 & 5.33 & \\
\hline \multicolumn{6}{|c|}{ SDS values according to Kawel-Boehm } \\
\hline LVM & 0.39 & 0.8 & -1.1 & 1.9 & 0.032 \\
\hline LVEDV & -0.53 & 1.0 & -2.5 & 1.6 & 0.015 \\
\hline LVESV & -0.03 & 1.3 & -2.0 & 3.7 & $\mathrm{~ns}$ \\
\hline $\mathrm{EF}$ & -0.25 & 1.6 & -5.2 & 2.0 & $\mathrm{~ns}$ \\
\hline SV & -0.76 & 1.1 & -3.1 & 1.4 & 0.002 \\
\hline CI & -1.11 & 2.0 & -3.6 & 1.0 & 0.001 \\
\hline
\end{tabular}

$L V M$ left ventricular mass, $B S A$ body surface area, $L V E D V$ left ventricular end diastolic volume, $L V E S V$ left ventricular end systolic volume, $E F$ ejection fraction, $C I$ cardiac index (cardiac output/BSA), $S V$ stroke volume. $S D$ standard deviation, $S D S$ SD score 
only two children $(8 \%)$ according reference values from Kawel-Boehm [28].

CMR-LVMI and CMR-LVM ${ }_{\mathrm{BSA}}$ were both correlated to annualized GFR decline after the examination, i.e., future kidney function (see Fig. 1, $r=-0.52, p=0.01$, and $r=-0.49, p=$ 0.02). This was not true for Echo-LVMI or Echo-LVM $\mathrm{BSA}_{\mathrm{BS}}$ (and only marginally for CMR-LVM SDS, $r=-0.40, p=$ 0.06). CMR and Echo-LVM were not correlated to present kidney function or to the slope of GFR decline prior to examination.

Increased CMR-LVM SDS was negatively correlated to aldosterone/renin ratio $(r=-0.63, p=0.02)$, which was not true for Echo-LVM-indices. Serum electrolytes, pH, PTH, vitamin D, and various markers of anemia correlated neither with CMRnor Echo-LVM measures. Lean children had higher cardiac output per BSA (cardiac index), with a correlation of CMR-CI SDS to body mass index (BMI) SDS of $r=-0.52(p=0.007)$.

\section{Agreement of left ventricular mass (LVM)}

As is evident from Tables 2 and 3, absolute values of LVM differed widely between the two groups with mean CMRLVM of $88.7 \pm 29.0$ vs. mean echo-LVM of $117.0 \pm 39.7$ $(p<0.0001)$. Even though the correlation between both measurements was high at $r=0.91(p<0.00)$, this is an imprecise measure of agreement, and Fig. 2 shows that the regression line is not parallel to the line of equivalence. The regression equation was Echo-LVM=6.1+CMR-LVM* 1.25 with $r^{2}=$ 0.84 ( $p$ of slope $<0.001, p$ of intercept 0.58 ).

The Bland-Altman plot (see Fig. 3) shows a mean difference between Echo- and CMR-LVM of $28.26 \pm 17.6 \mathrm{~g}$. Thus, the limits of agreement (mean $\pm 1.96 \times \mathrm{SD})$ were -6.2 to

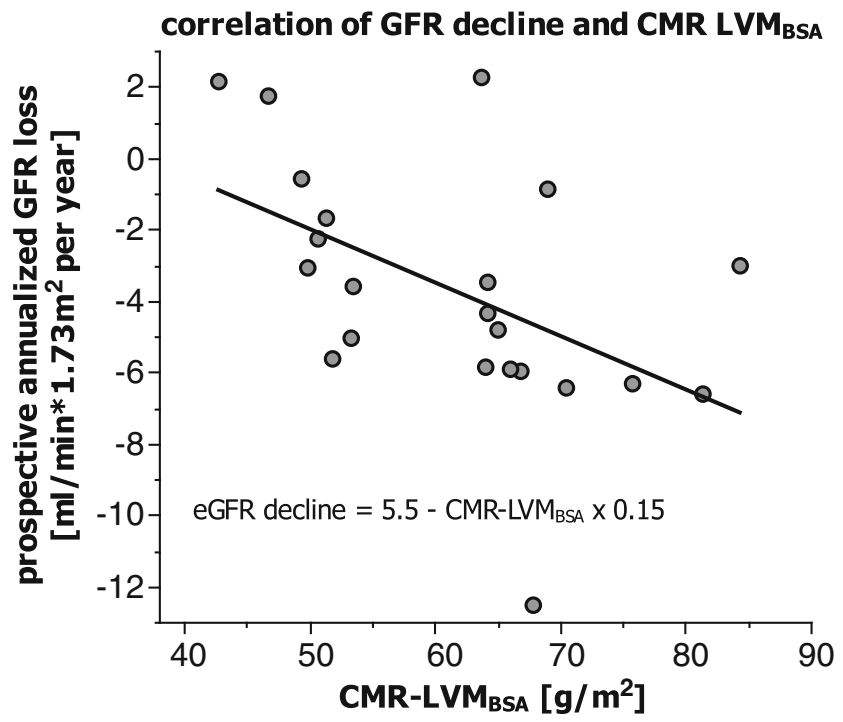

Fig. 1 Correlation of prospective annualized loss of estimated glomerular filtration rate (eGFR) with left ventricular mass (LVM) normalized to body surface area (BSA) measured by cardiac magnetic resonance (CMR). Solid line $=$ regression line correlation of left ventricular mass

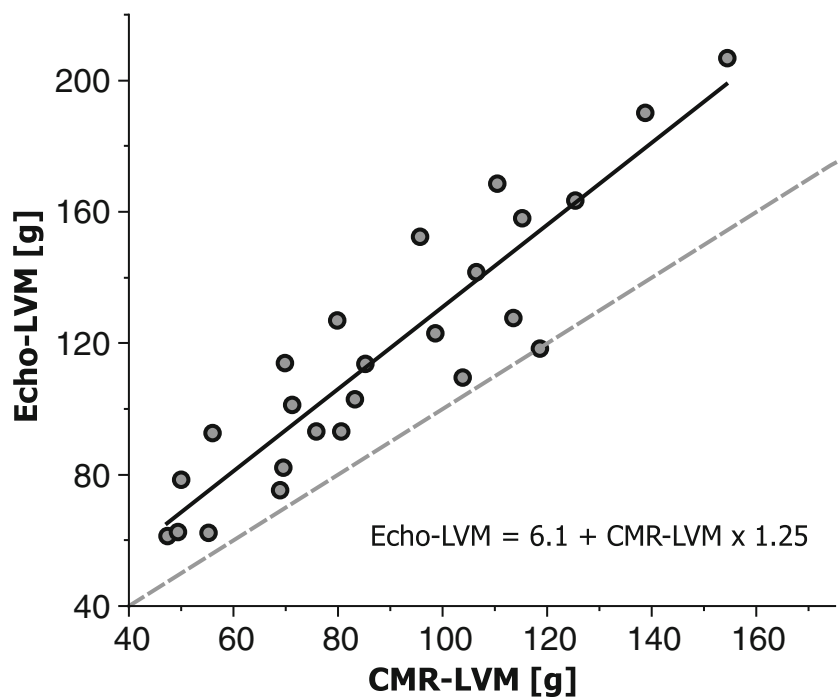

Fig. 2 Correlation of left ventricular mass (LVM) on echocardiography (Echo) and on cardiac magnetic resonance (CMR). Dotted line $=$ line of equivalence. Solid line $=$ regression line

$62.8 \mathrm{~g}$. The visual impression of the Bland-Altman plot suggests greater scatter at higher LVM measurements. This was confirmed by a significant correlation of the LVM difference to Echo-LVMI $(r=0.77, p<0.0001)$ but not CMR-LVMI $(r=$ $0.33, p=0.11$ ). The LVM measurement difference did not correlate to body dimensions, renal function, blood pressure, or anemia nor did it differ between males and females, pre- and post-transplantation or with stage of CKD.

However, the Echo-CMR difference of LVM was significantly higher in eight children treated for anemia with iron supplements or erythropoiesis stimulating agents $(40.3 \pm 18.5$

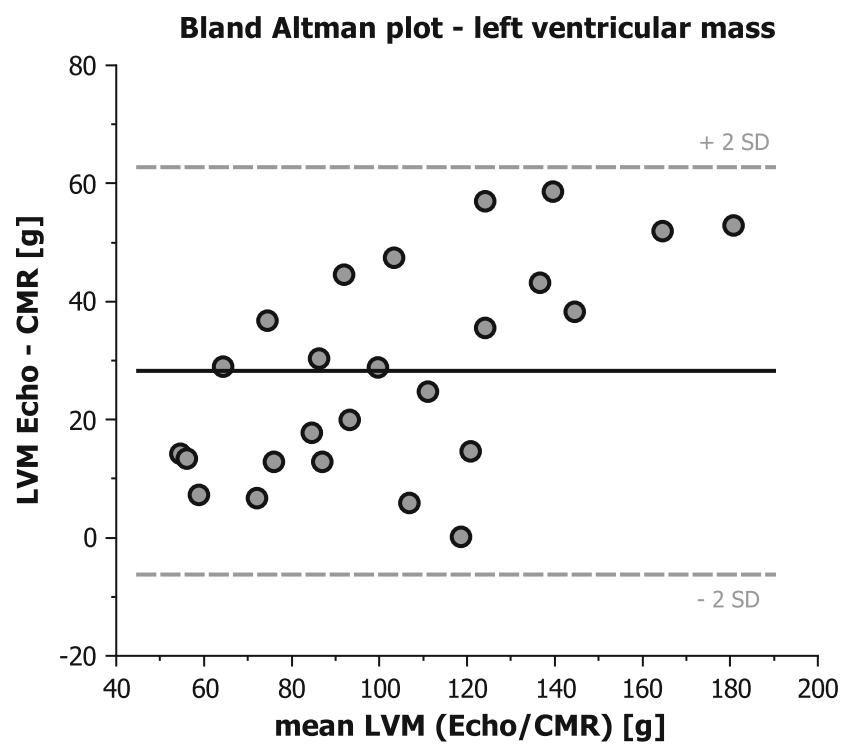

Fig. 3 Bland-Altman plot of the difference vs. the mean of left ventricular mass (LVM) measured by echocardiography (Echo) and cardiac magnetic resonance (CMR) 
vs. $22.6 \pm 14.5 \mathrm{~g}$ in 17 untreated patients, $p=0.016)$ and correlated to the dose of iron given $(r=0.42, p=0.036)$. There was no correlation to other markers of anemia such as hemoglobin, erythrocyte count, or mean corpuscular volume.

\section{Agreement of detecting left ventricular hypertrophy (LVH)}

The number of children with Echo-LVMI above the 95th percentile was much higher $(n=8)$, than those with CMR$\mathrm{LVM}_{\mathrm{BSA}}$ above the 95th percentile $(n=2)$ (see Table $4 \mathrm{a}$ ). Even though there was a stepwise increase of mean CMRLVM SDS from those with normal echocardiography $(n=5$, $0.03 \pm 0.8$ ), to concentric remodeling ( $n=12,0.33 \pm 0.86$ ), to concentric hypertrophy $(n=7,0.64 \pm 0.94)$ and to eccentric hypertrophy $(n=1,1.13)$, this did not reach statistical significance on ANOVA testing. Also, one of the two patients with CMR-LVH had a normal echocardiogram, however this patient's CMR-LVM ${ }_{\mathrm{BSA}}$ was only on the 96th percentile (SDS 1.76). Thus, the agreement between the two methods was only poor with a Cohen's kappa of 0.08 (95\% CL -0.23 to 0.40$)$. Presuming CMR to be the gold standard, echo had a poor specificity of $69.6 \%$ (CI $47-87 \%$ ) and poor sensitivity of $50 \%$ (95\% CI $1.3-98.7 \%)$. Only the negative predictive value was good with $94.12 \%$ (95\% CI 71.3-99.9\%), while the positive predictive value was expectedly low at $12.5 \%$ (95 \% CI 0.3-52.7\%).

To assess whether this disagreement was only due to the different indexing methods that are routinely used for EchoLVM (to height to the power of 2.7) and CMR-LVM (to BSA), we calculated the incidence of Echo-LVH defined as

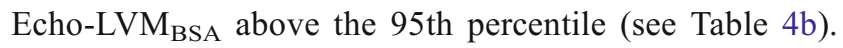
However, this only resulted in marginal improvement, with one less patient having Echo-LVH, resulting in a slightly better, but still poor Cohen's kappa of $0.11(95 \% \mathrm{CI}-0.24$ to 0.46). Accordingly, there was a slight increase of specificity

Table 4 Agreement of left ventricular hypertrophy (LVH) found on cardiac magnetic resonance (CMR) and echocardiography (Echo)

\begin{tabular}{|c|c|c|c|c|}
\hline & & \multicolumn{3}{|l|}{$\mathrm{CMR}^{-L_{V} M_{\mathrm{BSA}}}$} \\
\hline & & $>95$ th percentile & Normal & Total \\
\hline \multicolumn{5}{|c|}{ (a) Using standard indexing methods } \\
\hline \multirow[t]{3}{*}{ Echo-LVMI } & $>95$ th percentile & 1 & 7 & 8 \\
\hline & Normal & 1 & 16 & 17 \\
\hline & Total & 2 & 23 & 25 \\
\hline \multicolumn{5}{|c|}{ (b) Using indexing to body surface area (BSA) for both methods } \\
\hline \multirow[t]{3}{*}{ Echo-LVM $\mathrm{BSA}_{\mathrm{B}}$} & $>95$ th percentile & 1 & 6 & 7 \\
\hline & Normal & 1 & 17 & 18 \\
\hline & Total & 2 & 23 & 25 \\
\hline
\end{tabular}

LVMI left ventricular mass index
( $73.9 \%)$ and positive predictive value $(14.3 \%)$, with identical sensitivity and negative predictive value.

Figure 4 shows the individual differences between EchoLVMI percentile groups and CMR-LVM ${ }_{B S A}$ SDS score. Even though the Echo-LVMI percentiles were higher generally speaking, this was by no means true for all patients and there was considerable scatter.

\section{Agreement of ejection fraction (EF)}

Even though mean ejection fraction measured by echocardiography and CMR was similar (64.7 \pm 6.6 vs. $63.3 \pm 8, p=\mathrm{ns}$ ), correlation was worse than for LVM $(r=0.57, p=0.003$, see Fig. 5). The regression equation was Echo-EF= $36.3+$ CMR-EF $* 0.45 \quad(p=0.0003$ for intercept. $p=$ 0.003 for slope). The Bland-Altman plot shows a mean difference of 1.42 percentage points (limits of agreement: -12.6 to 15.4 ), with greater scatter at ejection fractions below $60 \%$ (see Fig. 6).

Thus, the agreement of detecting a reduced ejection fraction (below $55 \%$ ) was poor with a Cohen's kappa of 0.17 (95\% CI $-0.31-0.65)$; see Table 5. Assuming CMR to be the gold standard, echo had a good specificity of $90.5 \%$ and negative predictive value of $86.4 \%$, but a very poor sensitivity of $25 \%$ and positive predictive value of only $33 \%$.

As for LVM, the Echo-CMR difference of EF was significantly higher in children treated for anemia ( $\triangle \mathrm{EF} 5.71 \pm$ $6.8 \%$ vs. $-0.61 \pm 6.5 \%, p=0.036$ ) and correlated to the dose of iron given $(r=0.61, p=0.0013)$, but not to other markers of anemia.

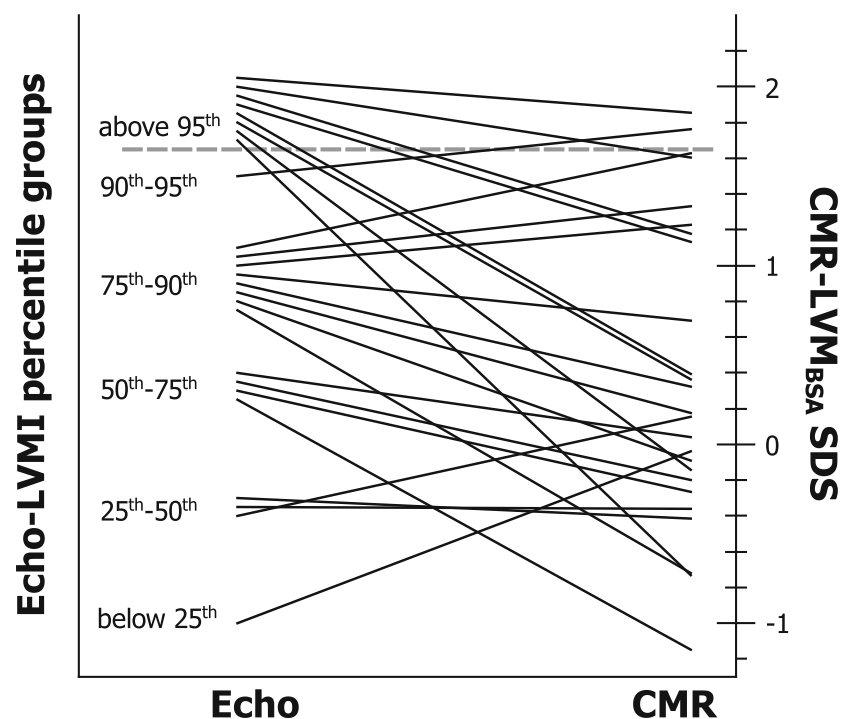

Fig. 4 Agreement of echocardiographic left ventricular mass index (Echo-LVMI) percentiles according to Khoury [11] with CMR-LVM $\mathrm{BSA}_{\mathrm{BA}}$ standard deviation score (SDS) according to Kawel-Boehm [28]. $C M R$ cardiac magnetic resonance, $L V M$ left ventricular mass 


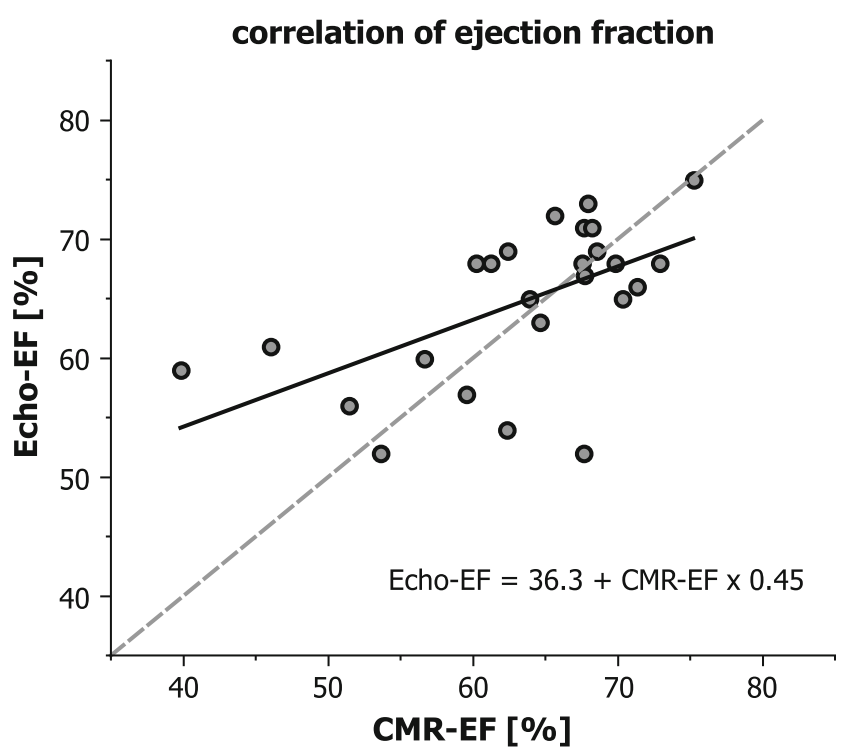

Fig. 5 Correlation of ejection fraction (EF) on echocardiography (Echo) and on cardiac magnetic resonance (CMR). Dotted line $=$ line of equivalence. Solid line $=$ regression line

\section{Comparison of patients pre- and post-kidney transplant (KTx)}

Patients with KTx had higher estimated GFR than those without ( $74 \pm 23$ vs. $\left.47 \pm 21 \mathrm{ml} / \mathrm{min}^{*} 1.73 \mathrm{~m}^{2}, p=0.005\right)$, but the two groups did not differ significantly with respect to age, gender, anthropometric measurements, anemia, ambulatory blood pressure or frequency, and intensity of antihypertensive treatment. The Echo-MRI differences in LVM, nLVM, and EF tended to be larger in patients with $\mathrm{KTx}$, but these differences were not significant. Cohen's kappa for agreement of LVH

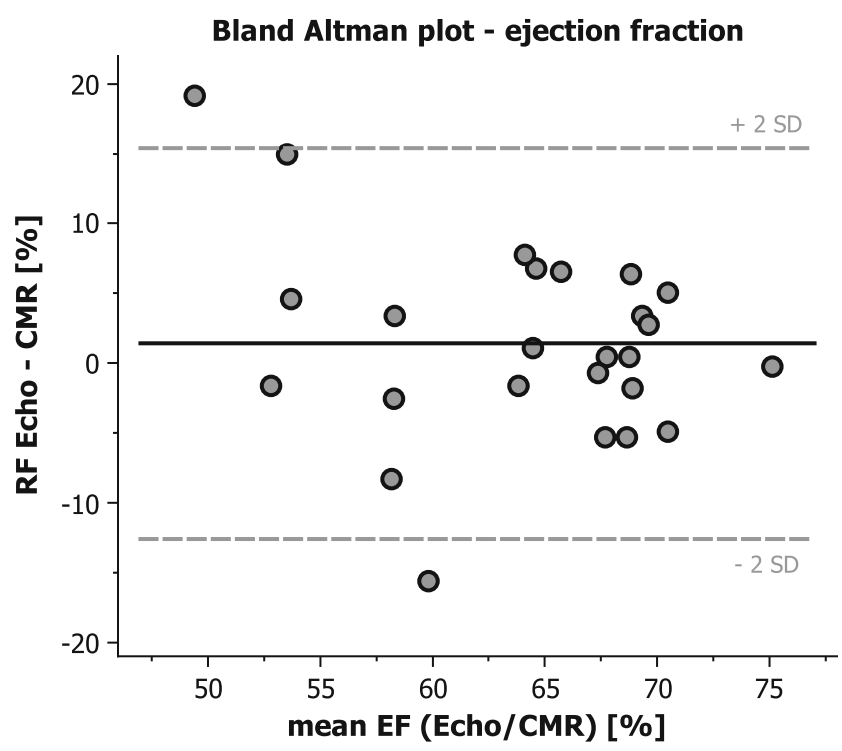

Fig. 6 Bland-Altman plot of the difference vs. the mean of ejection fraction (EF) measured by echocardiography (Echo) and cardiac magnetic resonance (CMR)
Table 5 Measurement of ejection fraction (EF) with echocardiography (Echo-) and cardiac magnetic resonance (CMR)

\begin{tabular}{lllll}
\hline & \multicolumn{3}{l}{ CMR-EF } & \\
\cline { 3 - 5 } & & $<55 \%$ & $>55 \%$ & Total \\
\hline Echo-EF & $<55 \%$ & 1 & 2 & 3 \\
& $>55 \%$ & 3 & 19 & 22 \\
& Total & 4 & 21 & 25 \\
\hline
\end{tabular}

classification was also worse in the KTx group, but $95 \%$ confidence intervals overlap widely $(0.12(-0.37$ to 0.1$)$ vs. $0.42(-0.17$ to 1$))$.

\section{Discussion}

With the improving prognosis of children with chronic kidney disease, increasing numbers of survivors are affected by severe cardiovascular disease starting in early adulthood [37]; thus, measuring left ventricular mass as a surrogate marker of cardiovascular disease is becoming clinically increasingly relevant in trying to prevent these changes. Echocardiography has been the standard technique for many years, but cardiac magnetic resonance offers an alternative which is promising, especially with regard to reliably measuring left ventricular volume independent of geometric assumptions, which may not hold for volume overloaded patients. Echo has been shown to overestimate CMR-LVM in adults with reduced renal function [19, 20] and hypertension [38, 39], but evidence is less clear in children [40]. While CMR is generally accepted as superior in quantifying LVM, the lack of validation with autopsy studies means it is not yet a solid gold standard. The aim of this study was to compare how well Echo and CMR agree in children with CKD and whether one method showed better correlation to renal parameters than the other.

On echocardiography, we find an incidence of left ventricular hypertrophy of $32 \%$, which is similar to previous studies, but with a higher proportion of concentric vs. eccentric changes compared to previous reports [1, 2, 41, 42]. However, adult CMR studies have suggested that dialysis patients have a unique kind of LVH, which is neither clearly concentric nor eccentric [43], which may be the cause of varying classification. The incidence of LVH was much lower on CMR with only $8 \%$. However, CMR-LVM SDS was still significantly increased from normal, confirming early cardiac changes found in previous studies $[17,18]$. Importantly, CMR-LVM and CMR-LVM $\mathrm{BSA}_{\mathrm{BA}}$ were able to predict GFR decline, which has not been found in any other pediatric echocardiographic studies. This relationship is plausible, as it has also been described in adult CKD patients [44]. As part of the cardiorenal syndrome, a small but significant influence of LVM on renal 
outcome is also found in studies in adult hypertensives $[45,46]$.

The comparison of LVM measurements by Echo and CMR showed a systematic difference between the two methods, where Echo-LVM was markedly higher than CMR-LVM, with an increasing difference at higher mean LVMs (see Figs. 2 and 3). As the difference did not correlate to body dimensions or CMR-LVM, but only to Echo-LVM, it can be postulated that it is due to a systematic error of the echo formula. Other authors have also found Echo-LVM to overestimate CMR-LVM, especially with reduced renal function [19, 20]. Indeed, the correlation of Echo-CMR-LVM difference to mean LVM found by Jakubovic et al. appears very similar to that in our study [20]. Absolute Echo-LVM differences only appear smaller in our study due to the smaller heart sizes in children. We also find that EchoCMR agreement of both LVM and EF was worse for patients treated for anemia, which may also relate to larger intravascular volume and consequently altered ventricular geometry in this group. To resolve this discrepancy, necropsy validated studies of CMR and Echo in patients with distorted geometry would be desirable.

On the other hand, comparing absolute LVM may justifiably be criticized as comparing "apples with oranges" as differences are inconsequential if application of correct reference values yields comparable results. We therefore also compared the classification of left ventricular hypertrophy using accepted standards (95th percentile cut-offs of LVMI for echo and $\mathrm{LVM}_{\mathrm{BSA}}$ for CMR), which still resulted in only poor agreement between the two methods with Cohen's kappa indicating agreement not significantly better than expected by chance. Applying identical indexing methods (i.e., 95th percentile of $\mathrm{LVM}_{\mathrm{BSA}}$ for both methods) this could only be marginally improved (see Table $4 \mathrm{a}$ and $\mathrm{b}$ ). Indexing LVM to BSA seems to underestimate the prevalence of hypertrophy especially in overweight patients [47], but while indexing to height 2.7 is more predictive of mortality in adult hemodialysis patients than indexing to BSA [48], it is still a matter of debate which method is superior in the general population $[49,50]$. In addition to the ongoing debate about the best way to index LVM [51], this matter is complicated further by the pediatric need for age-adjusted reference values (see supplementary table for an overview of available normal values). It is therefore unsurprising that the Echo-CMR agreement of assigning LVH is even poorer in our children than in adult CKD and hypertensive cohorts $[20,52]$.

With regard to cardiac function, CMR revealed a significant reduction of stroke volume, cardiac index, and end-diastolic volume in our cohort of CKD children, while ejection fraction was preserved. In a similar population a previous CMR study also found normal EF. [18]
Other functional cardiac studies on $\mathrm{CKD}$ children have employed various Echo techniques finding reduced fractional shortening and reduced EF, but both increased or decreased contractility [1, 3, 42, 53]. However, our comparison of Echo- and CMR-EF shows that agreement between both methods is far from optimal and especially poor in the clinically most relevant spectrum of EF below $60 \%$ (see Figs. 5 and 6), even though it was slightly better than in children with aortic insufficiency [54]. Thus there was only a poor Cohen's kappa of 0.17 for detecting reduced EF below $55 \%$. Measurement of other functional indices on Echo, such as cardiac output is difficult, as the estimation of stroke volume requires the velocity time integral of flow at left ventricular outflow tract. One functional study employing Echo and exercise testing found increased $\mathrm{CO}$ and $\mathrm{SV}$ in children with CKD and LVH compared to those with CKD without $\mathrm{LVH}$, however there was no comparison to normal children [55]. We too find a positive correlation of CMR$\mathrm{LVM}_{\mathrm{BSA}}$ to $\mathrm{SV}_{\mathrm{BSA}}(r=0.44, p=0.03)$, but not to $\mathrm{CO}$, however the overall SV and $\mathrm{CO}$ are decreased.

A CMR study in adult dialysis patients found decreased EF and increased EDV in adult dialysis patients, while EF was normal and EDV decreased in our pediatric non-dialysis population, which probably reflects more advanced stages of uremic cardiomyopathy in adults together with larger volume fluctuations under hemodialysis [43]. Other Echo studies in pediatric CKD have also found predominantly diastolic changes $[56,57]$, suggesting that impaired diastolic relaxation precedes systolic changes in uremic cardiomyopathy.

The current evidence does not justify the degradation of echocardiography, as it remains not only much easier and cheaper in clinical practice but also has the larger evidence base for outcome data so far. New developments such as 3D echocardiography show better correlation of LVM to CMR than 2D Echo even in children and may be suitable alternatives for clinical trials [58-60]. On the other hand, CMR is becoming increasingly available, protocols to measure LVM are relatively short ( $<30 \mathrm{~min})$ and do not require contrast medium and mass calculations are reproducible and less laborious with semi-automated tracing of ventricular outlines.

In summary, we find that Echo significantly overestimates LVM compared to CMR in children with CKD, which is consistent with previous findings from adults. The discrepancy between both methods is not easily removed by application of reference values, partly because a number of different reference populations and methods of indexing exist. The fact that CMR-LVM shows better prediction of GFR decline here suggests that it is worth further investigating the prognostic value of CMR vs. echocardiography. 
Acknowledgments The authors would like to thank Adriana Komancsek (radiographer) for the competent acquisition of CMR images.

Ethical approval The study confirmed to the declaration of Helsinki, was approved by the ethics committee of the University of Freiburg and registered in the German registry of clinical trials (Deutsches Register Klinische Studien, trial no. DRKS00003295).

Informed consent Prior written informed consent was obtained from all parents (and adolescents where appropriate).

Conflict of interest The authors declare no conflict of interest.

$\begin{array}{ll}\begin{array}{ll}\text { Glossary } \\ \text { BSA }\end{array} & \begin{array}{l}\text { Body surface area } \\ \text { CMR(-) }\end{array} \\ \text { Echo(-) } & \begin{array}{l}\text { Echocardiographetic resonance/measured by CMR } \\ \text { measured }\end{array} \\ \text { EF } & \text { Ejection fraction } \\ \text { LVM } & \text { Left ventricular mass } \\ \text { LVH } & \text { Left ventricular hypertrophy } \\ \text { LVMI } & \text { LVM indexed to height in } \mathrm{m}^{2.7} \\ \text { LVM } & \text { LVSA } \\ \text { ABPM } & \text { 24-hour ambulitory blood pressure measurement } \\ \text { CKD } & \text { Chronic kidney disease } \\ \text { CI } & \text { Confidence interval }\end{array}$

\section{References}

1. Mitsnefes MM, Kimball TR, Witt SA, Glascock BJ, Khoury PR, Daniels SR (2003) Left ventricular mass and systolic performance in pediatric patients with chronic renal failure. Circulation 107:864 868

2. Matteucci MC, Wühl E, Picca S, Mastrostefano A, Rinelli G, Romano C, Rizzoni G, Mehls O, de Simone G, Schaefer F, ESCAPE Trial Group (2006) Left ventricular geometry in children with mild to moderate chronic renal insufficiency. J Am Soc Nephrol 17:218-226

3. Chinali M, de Simone G, Matteucci MC, Picca S, Mastrostefano A, Anarat A, Caliskan S, Jeck N, Neuhaus TJ, Peco-Antic A, Peruzzi L, Testa S, Mehls O, Wühl E, Schaefer F (2007) Reduced systolic myocardial function in children with chronic renal insufficiency. $\mathrm{J}$ Am Soc Nephrol 18:593-598

4. Mitsnefes MM, Kimball TR, Border WL, Witt SA, Glascock BJ, Khoury PR, Daniels SR (2004) Impaired left ventricular diastolic function in children with chronic renal failure. Kidney Int 65:14611466

5. Ten Harkel ADJ, Cransberg K, Van Osch-Gevers M, Nauta J (2009) Diastolic dysfunction in paediatric patients on peritoneal dialysis and after renal transplantation. Nephrol Dial Transplant 24:19871991

6. Litwin M, Wühl E, Jourdan C, Trelewicz J, Niemirska A, Fahr K, Jobs K, Grenda R, Wawer ZT, Rajszys P, Tröger J, Mehls O, Schaefer F (2005) Altered morphologic properties of large arteries in children with chronic renal failure and after renal transplantation. J Am Soc Nephrol 16:1494-1500
7. Bilginer Y, Ozaltin F, Basaran C, Aki TF, Karabulut E, Duzova A, Besbas N, Topaloglu R, Ozen S, Bakkaloglu M, Bakkaloglu A (2007) Carotid intima-media thickness in children and young adults with renal transplant: internal carotid artery vs. common carotid artery. Pediatr Transplant 11:888-894

8. Doyon A, Kracht D, Bayazit AK, Deveci M, Duzova A, Krmar RT, Litwin M, Niemirska A, Oguz B, Schmidt BMW, Sözeri B, Querfeld U, Melk A, Schaefer F, Wühl E, 4C Study Consortium (2013) Carotid artery intima-media thickness and distensibility in children and adolescents: reference values and role of body dimensions. Hypertension 62:550-556

9. Devereux RB, Alonso DR, Lutas EM, Gottlieb GJ, Campo E, Sachs I, Reichek N (1986) Echocardiographic assessment of left ventricular hypertrophy: comparison to necropsy findings. Am J Cardiol 57:450-458

10. De Simone G, Devereux RB, Daniels SR, Koren MJ, Meyer RA, Laragh JH (1995) Effect of growth on variability of left ventricular mass: assessment of allometric signals in adults and children and their capacity to predict cardiovascular risk. J Am Coll Cardiol 25: 1056-1062

11. Khoury PR, Mitsnefes M, Daniels SR, Kimball TR (2009) Agespecific reference intervals for indexed left ventricular mass in children. J Am Soc Echocardiogr 22:709-714

12. Simpson JM, Savis A, Rawlins D, Qureshi S, Sinha MD (2010) Incidence of left ventricular hypertrophy in children with kidney disease: impact of method of indexation of left ventricular mass. Eur J Echocardiogr 11:271-277

13. Borzych D, Bakkaloglu SA, Zaritsky J, Suarez A, Wong W, Ranchin B, Qi C, Szabo AJ, Coccia PA, Harambat J, Mitu F, Warady BA, Schaefer F, International Pediatric Peritoneal Dialysis Network (2011) Defining left ventricular hypertrophy in children on peritoneal dialysis. Clin J Am Soc Nephrol 6:1934 1943

14. Schoenmaker NJ, van der Lee JH, Groothoff JW, van Iperen GG, Frohn-Mulder IME, Tanke RB, Ottenkamp J, Kuipers IM (2013) Low agreement between cardiologists diagnosing left ventricular hypertrophy in children with end-stage renal disease. BMC Nephrol 14:170

15. Grothues F, Smith GC, Moon JCC, Bellenger NG, Collins P, Klein HU, Pennell DJ (2002) Comparison of interstudy reproducibility of cardiovascular magnetic resonance with two-dimensional echocardiography in normal subjects and in patients with heart failure or left ventricular hypertrophy. Am J Cardiol 90:29-34

16. Armstrong AC, Gidding S, Gjesdal $\mathrm{O}, \mathrm{Wu} \mathrm{C}$, Bluemke DA, Lima JAC (2012) LV mass assessed by echocardiography and CMR, cardiovascular outcomes, and medical practice. JACC Cardiovasc Imaging 5:837-848

17. Schaefer B, Rusai K, Toth A, Pasti K, Ujszaszi A, Kreko M, Horvath E, Sallay P, Reusz GS, Merkely B, Tulassay T, Szabo AJ (2012) Cardiac magnetic resonance imaging in children with chronic kidney disease and renal transplantation. Pediatr Transplant 16: 350-356

18. Malatesta-Muncher R, Wansapura J, Taylor M, Lindquist D, Hor K, Mitsnefes M (2012) Early cardiac dysfunction in pediatric patients on maintenance dialysis and post kidney transplant. Pediatr Nephrol 27:1157-1164

19. Stewart GA, Foster J, Cowan M, Rooney E, McDonagh T, Dargie HJ, Rodger RS, Jardine AG (1999) Echocardiography overestimates left ventricular mass in hemodialysis patients relative to magnetic resonance imaging. Kidney Int 56:2248-2253

20. Jakubovic BD, Wald R, Goldstein MB, Leong-Poi H, Yuen DA, Perl J, Lima JA, Liu JJ, Kirpalani A, Dacouris N, Wald R, Connelly KA, Yan AT (2013) Comparative assessment of 2-dimensional echocardiography vs. cardiac magnetic resonance imaging in measuring left ventricular mass in patients with and without end-stage renal disease. Can J Cardiol 29:384-390 
21. Lorenz CH, Walker ES, Morgan VL, Klein SS, Graham TP (1999) Normal human right and left ventricular mass, systolic function, and gender differences by cine magnetic resonance imaging. J Cardiovasc Magn Reson 1:7-21

22. Vinnakota KC, Bassingthwaighte JB (2004) Myocardial density and composition: a basis for calculating intracellular metabolite concentrations. Am J Physiol Heart Circ Physiol 286:H1742H1749

23. Lai WW, Geva T, Shirali GS, Frommelt PC, Humes RA, Brook MM, Pignatelli RH, Rychik J, Task Force of the Pediatric Council of the American Society of Echocardiography, Pediatric Council of the American Society of Echocardiography (2006) Guidelines and standards for performance of a pediatric echocardiogram: a report from the task force of the pediatric council of the American Society of Echocardiography. J Am Soc Echocardiogr 19:1413-1430

24. De Simone G, Daniels SR, Kimball TR, Roman MJ, Romano C, Chinali M, Galderisi M, Devereux RB (2005) Evaluation of concentric left ventricular geometry in humans: evidence for agerelated systematic underestimation. Hypertension 45:64-68

25. Buechel EV, Kaiser T, Jackson C, Schmitz A, Kellenberger CJ (2009) Normal right- and left ventricular volumes and myocardial mass in children measured by steady state free precession cardiovascular magnetic resonance. J Cardiovasc Magn Reson 11:19

26. Robbers-Visser D, Boersma E, Helbing WA (2009) Normal biventricular function, volumes, and mass in children aged 8 to 17 years. J Magn Reson Imaging 29:552-559

27. Sarikouch S, Peters B, Gutberlet M, Leismann B, Kelter-Kloepping A, Koerperich H, Kuehne T, Beerbaum P (2010) Sex-specific pediatric percentiles for ventricular size and mass as reference values for cardiac MRI: assessment by steady-state free-precession and phase-contrast MRI flow. Circ Cardiovasc Imaging 3:65-76

28. Kawel-Boehm N, Maceira A, Valsangiacomo-Buechel ER, VogelClaussen J, Turkbey EB, Williams R, Plein S, Tee M, Eng J, Bluemke DA (2015) Normal values for cardiovascular magnetic resonance in adults and children. J Cardiovasc Magn Reson 17:29

29. Wühl E, Witte K, Soergel M, Mehls O, Schaefer F, German Working Group on Pediatric Hypertension (2002) Distribution of 24-h ambulatory blood pressure in children: normalized reference values and role of body dimensions. J Hypertens 20:1995-2007

30. Neuhauser HK, Thamm M, Ellert U, Hense HW, Rosario AS (2011) Blood pressure percentiles by age and height from nonoverweight children and adolescents in Germany. Pediatrics 127:e978-e988

31. Neuhauser H, Schienkiewitz A, Schaffrath Rosario A, Dortschy R, Kurth B-M (2013) Referenzperzentile für anthropometrische Maßzahlen und Blutdruck aus der Studie zur Gesundheit von Kindern und Jugendlichen in Deutschland (KiGGS) 2003-2006, 2nd edn. Robert Koch-Institut, Berlin

32. Bland JM, Altman DG (1986) Statistical methods for assessing agreement between two methods of clinical measurement. Lancet 1:307-310

33. Cohen J (1960) A coefficient of agreement for nominal scales. Educ Psychol Meas 20:37-46

34. Altman DG (1991) Practical statistics for medical research, 1st edn. Chapman and Hall, Oxford

35. Schwartz GJ, Brion LP, Spitzer A (1987) The use of plasma creatinine concentration for estimating glomerular filtration rate in infants, children, and adolescents. Pediatr Clin North Am 34:571-590

36. Filler G, Lepage N (2003) Should the Schwartz formula for estimation of GFR be replaced by cystatin C formula? Pediatr Nephrol 18: 981-985

37. Oh J, Wunsch R, Turzer M, Bahner M, Raggi P, Querfeld U, Mehls O, Schaefer F (2002) Advanced coronary and carotid arteriopathy in young adults with childhood-onset chronic renal failure. Circulation 106:100-105
38. Missouris CG, Forbat SM, Singer DR, Markandu ND, Underwood R, MacGregor GA (1996) Echocardiography overestimates left ventricular mass: a comparative study with magnetic resonance imaging in patients with hypertension. J Hypertens 14:1005-1010

39. Perdrix L, Mansencal N, Cocheteux B, Chatellier G, Bissery A, Diebold B, Mousseaux E, Abergel E (2011) How to calculate left ventricular mass in routine practice? An echocardiographic versus cardiac magnetic resonance study. Arch Cardiovasc Dis 104: 343-351

40. Barker PCA, Pasquali SK, Darty S, Ing RJ, Li JS, Kim RJ, DeArmey S, Kishnani PS, Campbell MJ (2010) Use of cardiac magnetic resonance imaging to evaluate cardiac structure, function and fibrosis in children with infantile Pompe disease on enzyme replacement therapy. Mol Genet Metab 101:332-337

41. Malikenas A, Cerniauskiene V, Jakutovic M, Jankauskiene A (2005) Left ventricular geometry in children with chronic renal failure. Med Kaunas Lith 41(Suppl 1):5-11

42. Raimondi F, Chinali M, Girfoglio D, Benincasa M, Pasquini L, Emma F, de Simone G, Chiara Matteucci M (2009) Inappropriate left ventricular mass in children and young adults with chronic renal insufficiency. Pediatr Nephrol 24:2015-2022

43. McGill RL, Biederman RWW, Getts RT, Hazlett SM, Sharma SB, Duran J, Brandys DE, Sysak JC, Sureshkumar KK, Sandroni SE, Marcus RJ (2009) Cardiac magnetic resonance imaging in hemodialysis patients. J Nephrol 22:367-372

44. Chen S-C, Su H-M, Hung C-C, Chang J-M, Liu W-C, Tsai J-C, Lin M-Y, Hwang S-J, Chen H-C (2011) Echocardiographic parameters are independently associated with rate of renal function decline and progression to dialysis in patients with chronic kidney disease. Clin J Am Soc Nephrol 6:2750-2758

45. Tsioufis C, Kokkinos P, Macmanus C, Thomopoulos C, Faselis C, Doumas M, Stefanadis C, Papademetriou V (2010) Left ventricular hypertrophy as a determinant of renal outcome in patients with high cardiovascular risk. J Hypertens 28:2299-2308

46. Andrikou E, Tsioufis C, Thomopoulos C, Andrikou I, Kasiakogias A, Leontsinis I, Kordalis A, Katsimichas T, Tousoulis D, Stefanadis C (2012) Left ventricular mass index as a predictor of new-onset microalbuminuria in hypertensive subjects: a prospective study. Am J Hypertens 25:1195-1201

47. Cuspidi C, Meani S, Negri F, Giudici V, Valerio C, Sala C, Zanchetti A, Mancia G (2009) Indexation of left ventricular mass to body surface area and height to allometric power of 2.7: is the difference limited to obese hypertensives? J Hum Hypertens 23: 728-734

48. Zoccali C, Benedetto FA, Mallamaci F, Tripepi G, Giacone G, Cataliotti A, Seminara G, Stancanelli B, Malatino LS, Investigators CREED (2001) Prognostic impact of the indexation of left ventricular mass in patients undergoing dialysis. J Am Soc Nephrol 12:2768-2774

49. Cuspidi C, Facchetti R, Bombelli M, Sala C, Tadic M, Grassi G, Mancia G (2015) Prognostic value of left ventricular mass normalized to different body size indexes: findings from the PAMELA population. J Hypertens 33:1082-1089

50. De Simone G, Kizer JR, Chinali M, Roman MJ, Bella JN, Best LG, Lee ET, Devereux RB, Strong Heart Study Investigators (2005) Normalization for body size and population-attributable risk of left ventricular hypertrophy: the strong heart study. Am J Hypertens 18: 191-196

51. Foppa M, Duncan BB, Rohde LEP (2005) Echocardiographybased left ventricular mass estimation. How should we define hypertrophy? Cardiovasc Ultrasound 3:17

52. Armstrong AC, Gjesdal O, Almeida A, Nacif M, Wu C, Bluemke DA, Brumback L, Lima JAC (2014) Left ventricular mass and hypertrophy by echocardiography and cardiac magnetic resonance: the multi-ethnic study of atherosclerosis. Echocardiography $31: 12-20$ 
53. Simpson JM, Rawlins D, Mathur S, Chubb H, Sinha MD (2013) Systolic and diastolic ventricular function assessed by tissue Doppler imaging in children with chronic kidney disease. Echocardiography 30:331-337

54. Ley S, Eichhorn J, Ley-Zaporozhan J, Ulmer H, Schenk J-P, Kauczor H-U, Arnold R (2007) Evaluation of aortic regurgitation in congenital heart disease: value of MR imaging in comparison to echocardiography. Pediatr Radiol 37:426-436

55. Weaver DJ, Kimball TR, Koury PR, Mitsnefes MM (2009) Cardiac output and associated left ventricular hypertrophy in pediatric chronic kidney disease. Pediatr Nephrol 24:565-570

56. Lindblad YT, Axelsson J, Balzano R, Vavilis G, Chromek M, Celsi G, Bárány P (2013) Left ventricular diastolic dysfunction by tissue Doppler echocardiography in pediatric chronic kidney disease. Pediatr Nephrol 28:2003-2013

57. Dogan CS, Akman S, Simsek A, Ozdem S, Comak E, Gokceoglu AU, Kardelen F, Koyun M (2015) Assessment of left ventricular function by tissue Doppler echocardiography in pediatric chronic kidney disease. Ren Fail. doi:10.3109/0886022X.2015.1061301
58. Lu X, Xie M, Tomberlin D, Klas B, Nadvoretskiy V, Ayres N, Towbin J, Ge S (2008) How accurately, reproducibly, and efficiently can we measure left ventricular indices using M-mode, 2-dimensional, and 3-dimensional echocardiography in children? Am Heart J 155:946-953

59. Pacileo G, Castaldi B, Di Salvo G, Limongelli G, Rea A, D'Andrea A, Russo MG, Calabrò R (2013) Assessment of left-ventricular mass and remodeling in obese adolescents: M-mode, 2D or 3D echocardiography? J Cardiovasc Med 14:144-149

60. Friedberg MK, Su X, Tworetzky W, Soriano BD, Powell AJ, Marx GR (2010) Validation of 3D echocardiographic assessment of left ventricular volumes, mass, and ejection fraction in neonates and infants with congenital heart disease: a comparison study with cardiac MRI. Circ Cardiovasc Imaging 3:735742

61. Foster BJ, Mackie AS, Mitsnefes M, Ali H, Mamber S, Colan SD (2008) A novel method of expressing left ventricular mass relative to body size in children. Circulation 117: 2769-2775 